\title{
Neutron and Photon Research for Texture and Stress Characterisation of Advanced Materials
}

\author{
H.-G. Brokmeier ${ }^{1,2, a}$ \\ ${ }^{1}$ Institute of Materials Science and Engineering, TU Clausthal, Agricolastr.6, D-38678 \\ Clausthal-Zellerfeld, Germany \\ ${ }^{2}$ GKSS Research Center Geesthacht GmbH, Max- Planck-Str. 1, D-21502 Geesthacht, Germany \\ aheinz-guenter.brokmeier@tu-clausthal.de
}

Keywords: Neutron diffraction, Synchrotron radiation, Crystallographic texture, Stress analysis.

\begin{abstract}
Worldwide materials science diffractometers at large scale facilities were built recently to improve experimental options for the characterization of advanced materials. Thermal neutrons as well as hard X-rays have a relatively high penetration power that non-destructive investigations of stress profiles and texture gradients are possible. Due to the main difference between neutrons and photons, which is the brilliance of the beam, the gage volume of synchrotron experiments is much smaller than with neutrons. That means, according to the material itself local resolution in mm-scale is preferred by neutrons and in $\mu \mathrm{m}$ scale by synchrotron radiation. The microstructure of laser welded Al shows fine grained parts were synchrotron radiation can be used while coarse grained parts need neutrons for better grain statistics. Both radiations can also be used to perform in situ experiments for stress and texture analysis. A combination of neutron and synchrotron measurements was used to explain the texture influence on the activation of twinning during $\mathrm{Mg}$-extrusion. Neutron diffractometers, such as Stress-Spec@FRM II/Garching-Germany, or synchrotron diffractometers, such as Harwi-II@Haslab/Hamburg-Germany, are excellent for materials characterization in combination with electron diffraction and laboratory X-ray diffraction.
\end{abstract}

\section{Introduction}

The advantages of materials science investigations using neutron or synchrotron radiation is based on there unique physical properties of this two radiations [1]. In the case of crystallographic texture analysis and residual stress determination the high penetration power of thermal neutrons and hard $\mathrm{X}$-rays are of basic interest [2]. Non-destructive investigations of engineering materials as well as in situ experiments are possible in a reasonable time scale. Figures $1 \mathrm{a}$ and $1 \mathrm{~b}$ show typical examples with a welded sample of $30 \mathrm{~cm}$ in length mounted on synchrotron diffractometer and a compression sample mounted in a loading device at a neutron diffractometer.

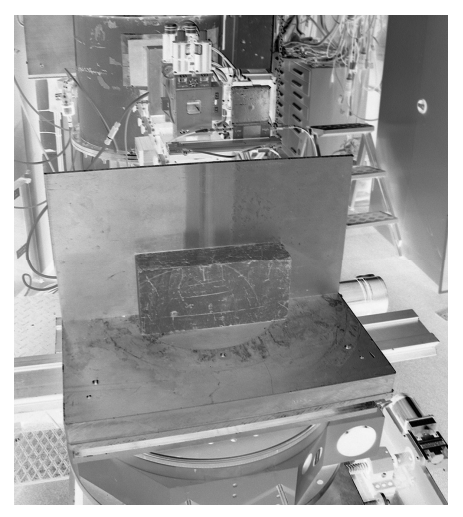

Fig.1a: Laser welded Al-plates (total length $30 \mathrm{~cm}$ )

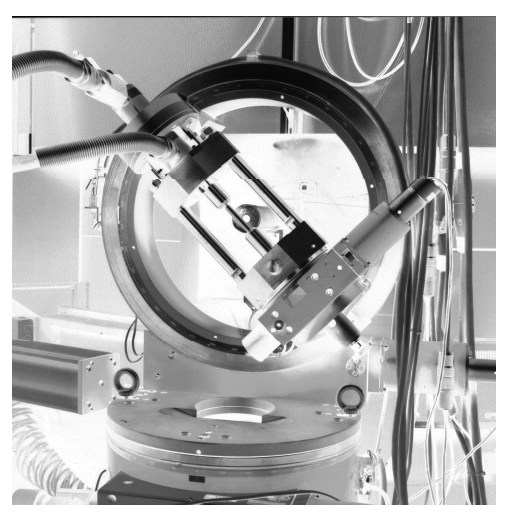

Fig.1b: Loading device with a compression sample mounted in a Eulerian cradle 
Crystallographic texture, microstructure and residual stress are among others important informations of polycrystalline materials related to the same grain arrangement. That means to understand materials properties in all details from processing to application and recycling connected with energy and cost efficiency one needs all the above informations. The orientation stereology as proposed by Bunge using EBSD [3] allows a quantitative 3D texture description related to $\mathrm{x}, \mathrm{y}, \mathrm{z}$ coordinates combining with a microstructure investigation. 3D X-ray Diffraction Microscopy (3DXRD) [4] or X-ray diffraction contrast tomography (DCT) [5] using synchrotron radiation for the mapping of single grains. The present paper focuses on the investigation of bulk samples to map stress and texture gradients of bulk samples or to carry out in situ experiments. Considering new light weight structures and dissimilar welded materials property gradients are of great importance.

The information density as shown for example for a synchrotron measurement of about 1 sec. includes all what is connected with reflection intensities, peak positions and peak profiles (fig. 2). In addition to new instrumentation a fast data analysis has been developed to handle large amounts of pattern.

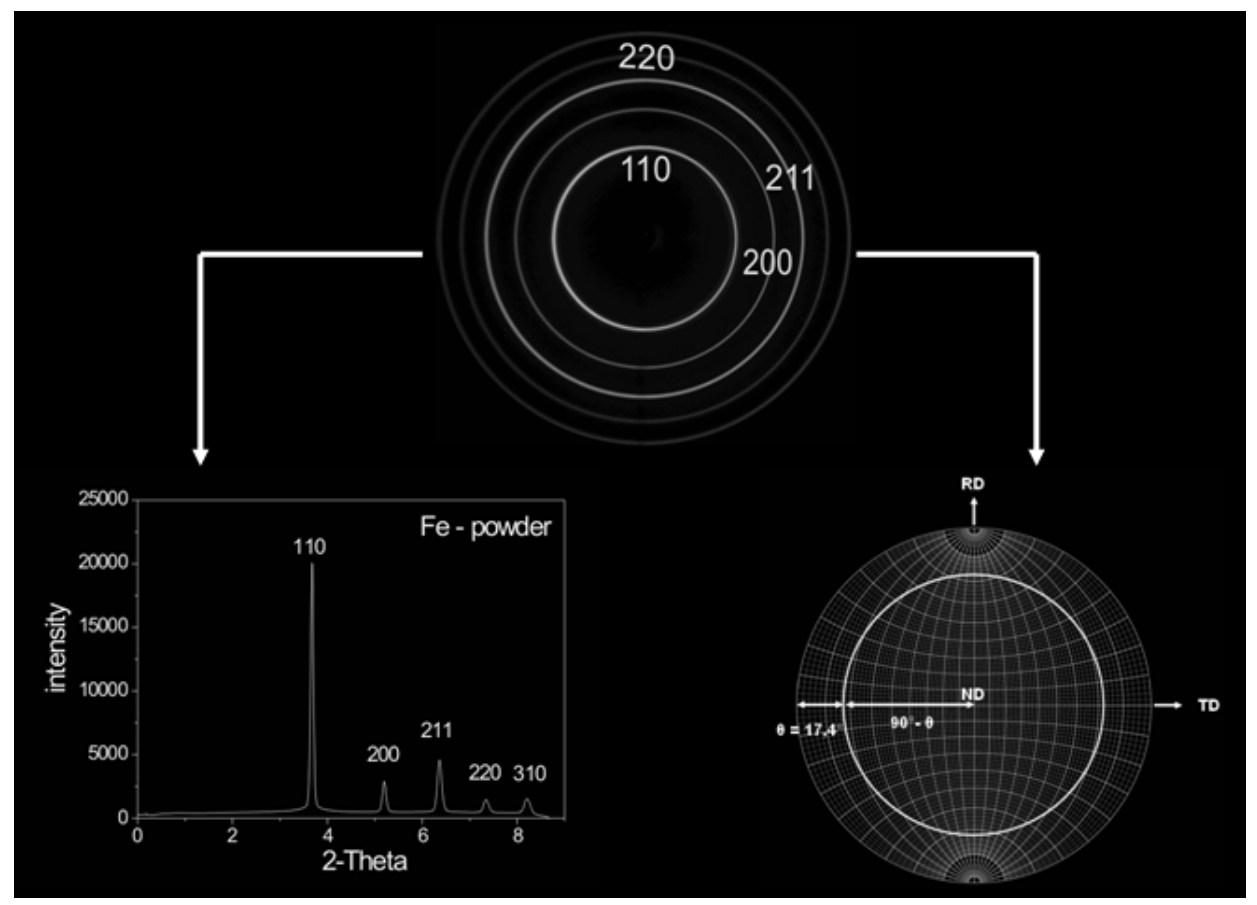

Fig. 2: Area detector picture measured with hard X-rays and data extraction for diffraction pattern analysis (strain, phase analysis, thermal expansion coefficients, and lattice dependent elastic constants) and data extraction for crystallographic textures (right).

Typical local resolutions are gage volumes between $1 \mathrm{~mm}^{3}-10 \mathrm{~mm}^{3}$ for neutrons and $0.1 \mathrm{~mm}^{3}$ $-1 \mathrm{~mm}^{3}$ for hard X-rays.

\section{Investigation of welded specimens}

Welding is one of the key technologies of the challenges of the $21^{\text {st }}$ century. Development of new materials for light weight constructions, energy technologies and biomaterials require among others the characterization of the anisotropy of the materials properties. The present example of laser beam welding of A15083 with A16013 [6], which is part of an intense study on laser welding, friction welding and friction stir welding of light weight materials, was investigated by thermal neutrons at TEX-2 diffractometer/FRG-1 Geesthacht, Germany, by hard X-rays using BW5 and Harwi-II at Haslab/Hamburg and by electron diffraction.

The combination of these methods was necessary because of the inhomogeneity of the microstructure over the weld. Particular recrystallization leads to coarse graining in the welded zones 
as well as in both heat affected zones. To give only one example of the whole investigation the anisotropy of the e-modulus over the weld is presented in Figure 3. The data were calculated from single crystal properties and the orientation distribution function as described by Bunge [6]. One can see on one hand the differences in the basic materials due to the alloying elements and the pre-processing. On the other hand one can see also that the weld itself has quite a different behaviour than the two base materials (Fig. 3a). A little stronger anisotropy was observed in the head affected zone where long elongated grains dominate the microstructure (Fig. 3b).

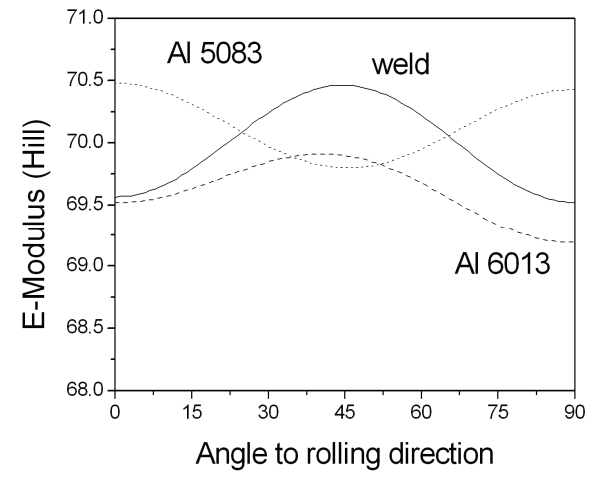

Fig. 3a: E-Modulus A16013 - weld - A15083

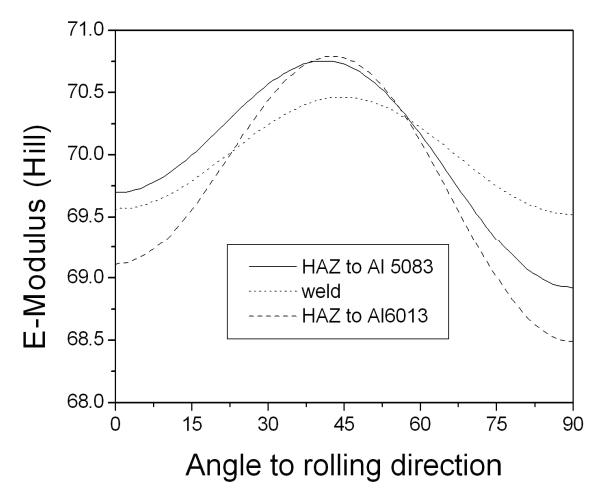

Fig. 3b: E-Modulus along HAZ - weld - HAZ

In parallel to this investigation stress profile over the weld, micro hardness and crystallographic phase evolution have been studied.

\section{In situ investigation of twinning in Mg alloys}

Hexagonal materials such as Mg-, Ti- or Zr-alloys are of basic interest for many different applications. Due to the crystal structure and the multi-phase alloys $(\alpha-\mathrm{Ti}+\beta-\mathrm{Ti})$ deformation behaviour and materials properties is more complicated than for cubic alloys. An essential part in the deformation behaviour of $\mathrm{Mg}$-alloys is the need to combine different deformation modes (glide systems plus twinning). In situ loading experiments interpreted by the simulation of the texture development contribute to understand the influence of the initial texture on the activation of glide systems. The present example is also a combination of bulk texture measurements by neutron diffraction, in situ loading experiments by hard X-rays and conventional mechanical tests of a Mg-AM20 alloy [7].

Neutron diffraction is ideal for the characterization of tensile samples in a non-destructive way. This allows subsequent thermo-mechanical treatments as well as loading tests on identical samples. For different rectangular extruded $\mathrm{Mg}$-alloys tensile samples with loading axis perpendicular, parallel and $45^{\circ}$ to extrusion direction were prepared. In an ex situ experiment, the crystallographic texture averaged over the whole cross section of $6 \mathrm{~mm}$ thickness was measured before tension and after failure.

Hard X-rays available at the high energy beamlines BW5 or Harwi-II at Haslab/Hamburg, Germany have been used for in situ investigations. The crystallographic texture was measured under load with $0 \%, 2.5 \%, 6 \%, 12 \%, 19 \%$ and $22.5 \%$. Due to the short wavelength of the hard X-rays of about $0.124 \AA$ the image plate area detector collects complete Debye Scherrer cones of about 6 Mg-reflections simultaneously. One needs only a sample rotation to get sufficient pole figure informations to be able to calculate the orientation distribution function (ODF). The beam size of the $100 \mathrm{keV}$ synchrotron beam was $1 \times 1 \mathrm{~mm}^{2}$. Recalculated pole figures before tension and after $2.5 \%$ strain are shown in Figure 4. Data interpretation was done by comparing the experimental texture evolution under applied load with texture simulations carried out by the VPSC (visco-plastic-self-constant) method [8]. In Figure one can see the dynamic evolution of the relative activities for different glide systems and twinning. The deformation of this sample starts with strong 
basal slip plus tensile twinning. During continuous strain tensile twinning is less important and prismatic as well as pyramidal a slip became more important.

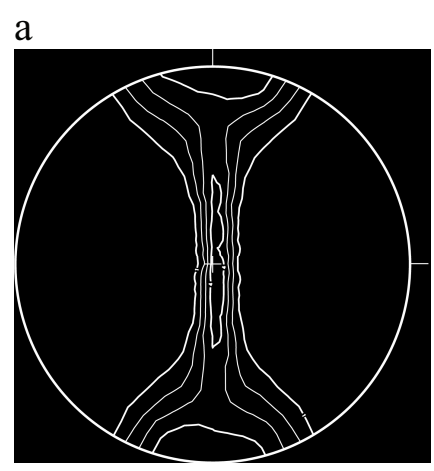

(0002)

Fig. 4a: Mg-AM20 before tension b

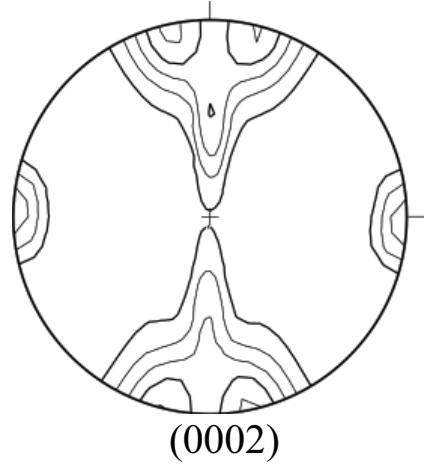

Fig. 4b: Mg-AM20 after $2.5 \%$ strain

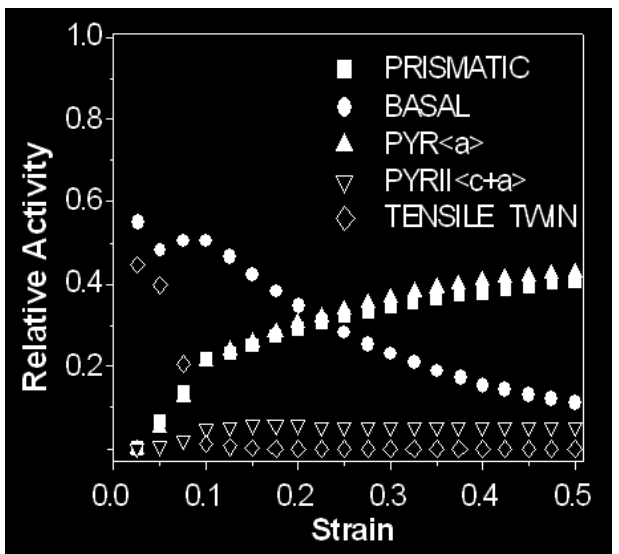

Fig. 5: Slip system activation during tensile test

\section{Summary}

Firstly, it has to be pointed out that neutrons as well as photons contribute in an excellent manner to the development and characterization of advanced materials and applications. Secondly, neutrons and photons are complementary so that bulk investigations are favoured by neutrons and local investigations in $\mu \mathrm{m}$ to $\mathrm{mm}$ scale as well as fast in situ measurements are excellent for synchrotron radiation. Thirdly, advanced materials in new constructions are characterized by gradient properties, which can be controlled in profile investigations of microstructure, strain and crystallographic texture by a combination of methods using electron, X-ray neutron and synchrotron diffraction. Fourthly, non-destructive investigations in welded samples and under specific conditions (high temperature or applied load) can contribute for basic understanding of deformation, recovery and recrystallization.

\section{References}

[1] W. Reimers, A.R. Pyzalla, A. Schreyer, H. Clemens: Neutrons and Synchrotron Radiation in Engeneering Materials Science (Wiley-VCH Germany 2008).

[2] H.-G. Brokmeier: Physica B: Condensed Matter Vol. 385-386 (2006) p. 623.

[3] H. J. Bunge: Advanced Engineering Materials Vol. 3 (2001) p. 25.

[4] H. F. Poulsen, W. Ludwig and S. Schmidt in: Neutrons and Synchrotron Radiation in Engeneering Materials Science edited by W. Reimers, A.R. Pyzalla, A. Schreyer, H. Clemens, chapter 19, Wiley-VCH Germany 2008.

[5] A. King, M. Herbig, W. Ludwig, P. Reischig, E.M. Lauridsen, T. Marrow and J.Y. Buffière: Nucl. Instrum. and Methods in Phyiscs Research B Vol 268 (2010) p. 291.

[6] H.-G. Brokmeier, S. Lenser, R. Schwarzer, V. Ventzke, S. Riekehr, M. Kocak and J. Homeyer: Materials Science Forum Vol. 539-543 (2007) p. 3894.

[7] S. B.Yi, H.-G. Brokmeier, R.E. Bolmaro, K.U. Kainer and T. Lippmann: Scripta Mat. Vol 51 (2004) p. 455.

[8] R.A. Lebensohn, C.N. Tome; Acta Metall Mater Vol. 41 (1993) p. 2611. 
Advances in Superalloys

doi:10.4028/www.scientific.net/AMR.146-147

Neutron and Photon Research for Texture and Stress Characterisation of Advanced Materials

doi:10.4028/www.scientific.net/AMR.146-147.891 